\title{
Downregulation of miR-95-3p inhibits proliferation, and invasion promoting apoptosis of glioma cells by targeting CELF2
}

\author{
BO FAN ${ }^{1}$, BAO-HUA JIAO ${ }^{1}$, FENG-SHI FAN ${ }^{2}$, SHENG-KUI LU $^{1}$, JIAN SONG $^{1}$, \\ CHENG-YONG GUO ${ }^{1}$, JIAN-KAI YANG ${ }^{1}$ and LIANG YANG ${ }^{1}$ \\ ${ }^{1}$ Department of Neurosurgery, The Second Affiliated Hospital of Hebei Medical University, Shijiazhuang, Hebei 050000; \\ ${ }^{2}$ Department of Neurosurgery, The First Affiliated Hospital of Hebei Medical University, \\ Shijiazhuang, Hebei 050030, P.R. China
}

Received April 15, 2015; Accepted May 25, 2015

DOI: $10.3892 /$ ijo.2015.3080

\begin{abstract}
Gliomas are the most common and aggressive types of tumors in human brain, of which the prognosis remains dismal because of their biological behavior. The involvement of miRNAs in tumorigenesis of various kinds of cancers drives us to explore new miRNAs related to gliomas. We measured expression level of miR-95-3p by qRT-PCR in human glioma and non-neoplasm brain tissues and found that higher level of miR-95-3p in glioma tissues of higher grade. Biological functions of miR-95-3p on glioma cells were investigated by MTT assay, flow cytometry and transwell assay. We discovered the cell lines transfected with miR-95-3p ASO (antisense oligonucleotide) had retarded proliferation and invasion but enhanced apoptosis ability. We searched on-line tool Targetscan and selected CELF (CUGBP- and ETR-3-like family 2) as a putative target. Luciferase reporter was employed to confirm the binding sites in $3^{\prime} \mathrm{UTR}$ region of CELF2 for miR-95-3p. The correlation between expression of CELF2 and miR-95-3p was determined by western blotting and qRT-PCR both in cell lines and human samples. Results showed CELF2 was a direct target of miR-95-3p and expression levels of CELF2 and miR-95-3p were negatively correlated. Finally, CELF2 largely abrogated the effects of miR-95-3p on proliferation, invasion and apoptosis of glioma cells in rescue experiments, which verified the role of CELF2 in miR-95-3p regulating glioma biological behavior. In conclusion, our data suggest the expression level of miR-95-3p is positively related to glioma grade and downregulation of miR-95-3p affects proliferation, invasion and apoptosis of glioma cells by targeting CELF2. We identified miR-95-3p as a putative therapeutic target and CELF2 as a potential tumor suppressor.
\end{abstract}

Correspondence to: Professor Bao-Hua Jiao, Department of Neurosurgery, The Second Affiliated Hospital of Hebei Medical University, 215 Hepingxi Road, Shijiazhuang, Hebei 050000, P.R. China E-mail: jiaobh2000@163.com

Key words: miR-95-3p, CUGBP- and ETR-3-like family 2, proliferation, invasion, apoptosis, glioma

\section{Introduction}

Gliomas are the most common and aggressive types of tumors in central nervous system. It has been proved that dozens of molecules and signal pathways are involved in the genetic alterations and chromosome aberrations during tumorigenesis. The abnormal abilities of invasion, proliferation, migration and apoptosis of glioma cells are the main obstacles to better prognosis (1-3). Despite the achievements in conventional treatments, the median survival period of patients suffering from glioblastoma multiforme (GBM), a type of grade IV glioma, remains at 12 months over the past few decades (4). Hence, discovering new therapeutic targets and developing mechanism-based approaches is an urgent need.

MicroRNAs (miRNA or miR) are a group of endogenous non-coding small RNAs which are expressed in the vast majority of eukaryotes (5). They cause gene silencing by targeting specifically 3'-untranslated regions (3'UTR) of mRNAs, leading to the degradation or repression of mRNA translation $(6,7)$. Some miRNA precursors can form two stably expressing mature miRNAs, which are distinguished by adding ' $-3 p$ ' or ' $-5 p$ ' to the end of the name according to their procession positions. Emerging evidence has strongly indicated that this post-transcriptional regulation by miRNAs has major implications in many physiological and pathological processes, and is associated with the initiation and progression of human cancer, including glioma (8-11). Some miRNAs are proved to be crucial oncogenes or tumor suppressors of glioma (12-14).

miR-21, miR-10b, miR-93 and miR-155 are upregulated in gliomas and have been shown to be significant contributors in tumor growth (13,15-17); while miR-7, miR-124, miR-128 and miR-137 are downregulated with tumor suppressor activities (16,18-20). Diverse expression profiles between non-neoplasm and glioma tissues can partly elucidate the functions of miRNAs in tumorigenesis and therefore can be utilized to predict novel therapeutic targets. We noticed that miR-95 was reported to have different expression profiles between glioma tissues and non-neoplasm brain tissues by sequencing analysis (21). The results of functional research on miR-95 seem to be contradictory, as miR-95 promotes the growth of colorectal, pancreatic, prostate and breast cancer 
(22-24), while has anticancer activity in hepatic, brain and neck cancer $(25,26)$. However, miR-95 has not been previously reported in gliomas. So here we recruited miR-95-3p as a candidate to explore its biological function in glioma.

In this study, we first compared the expression level of miR-95-3p in glioma and non-neoplasm brain tissues by qRT-PCR, and evaluated its influence on the proliferation, invasion, cell cycle and apoptosis of glioma in vitro. We discovered the reverse relationship between CELF2 and miR-95-3p expression both in cell lines and human samples, and utilized luciferase reporter assay to confirm that CELF2 was a novel target of miR-95-3p. Rescue experiment showed that downregulation of CELF2 could largely abolish the effect induced by repression of miR-95-3p. Taken together, we conclude that miR-95-3p affects proliferation, apoptosis and invasion of glioma cells by targeting CELF2, and we identified miR-95-3p as a putative therapeutic target and CELF2 as a potential tumor suppressor.

\section{Materials and methods}

Clinical sample collection. Human glioma samples and non-neoplasm brain samples were collected from the Neurosurgery Department of the Second Affiliated Hospital of Hebei Medical University in 2014. Fifteen females and 35 males were included, with ages ranging from 30 to 60 . Thirty-five tumor samples, including 4 grade I, 5 grade II glioma, 17 grade III and 9 grade IV tissues (GBM), were obtained by surgical resection, and 5 non-neoplasma samples as control were obtained by intracranial decompression for severe traumatic brain injury. Diagnoses were established histologically by two pathologists according to the WHO classification guidelines. All samples were stored at $-80^{\circ} \mathrm{C}$ in liquid nitrogen immediately after resection. Written informed consent was obtained from all patients and the study was approved by the ethics committee of The Second Affiliated Hospital of Hebei Medical University.

Cell culture and transfection. Human glioma cell lines U87 and U251 were obtained from the American Type Culture Collection (Manassas, VA, USA) and were maintained in $\alpha$-MEM (Gibco BRL, Grand Island, NY, USA) supplemented with $10 \%$ fetal bovine serum (FBS), $100 \mathrm{U} / \mathrm{ml}$ penicillin and $100 \mu \mathrm{g} / \mathrm{ml}$ streptomycin at $37^{\circ} \mathrm{C}$ with $5 \% \mathrm{CO}_{2}$. The miR-95-3p ASO, control ASO, CELF2 siRNA and control siRNA were synthesized and purified by GenePharma, Shanghai, China. The RNA sequences mentioned above were as follows: miR-95-3p ASO: 5'-TGCTCAATAAATACCCGTTGAA-3'; Ctrl ASO: 5'-TTATCGCCATGTCCAATGAGGCT-3'; CUGBP2 siRNA: 5'-GCAAACCUUACUGAUCCUA-3'; Ctrl siRNA: 5'-UAAGGCUAUGAAGAGAUAC-3'.

The control RNAs contain random sequences and are predicted not to have any interactions in cells. Cell transfection was performed using Lipofectamine 2000 (Invitrogen, Carlsbad, CA, USA) according to the manufacturer's instructions. To transfect RNA oligonucleotides, $10 \mathrm{pmol}$ of miRNA antisense oligonucleotides and ctrl ASO were used. For rescue experiments, cells were contransfected with $10 \mathrm{pmol}$ of miRNA ASO and siRNA or control siRNA in a 96-well plate.
$R N A$ extraction and real-time quantitative $R T-P C R$. Total cell RNA was extracted using TRIzol reagent (Invitrogen) according to the manufacturer's instructions. miRNA level was measured by qRT-PCR. All the primers of miRNAs for TaqMan miRNA assays were purchased from Genewiz (Beijing, China). Human U6 small nuclear RNA was used as an internal normalized reference. The forward and reverse primers of miR-95-3p and U6 are as follows: miR-95-3p F/R, 5'-TGCGGTTCAACGGGTATTTATTG-3'/5'-CCAGTG CAGGGTCCGAGGT-3'; U6 F/R, 5'-TGCGGGTGCTCGCTT CGGCAGC-3'/5'-CCAGTGCAGGGTCCGAGGT-3'. RNA $(5 \mu \mathrm{g})$ was mixed with $1 \mu \mathrm{l}$ of MMLV reverse transcriptase (Promega, Madison, WI, USA) to synthesize complementary DNA according to the manufacturer's instructions. The expression of miR-95-3p in human samples was quantified using SYBR ${ }^{\circledR}$ Premix Ex Taq ${ }^{\mathrm{TM}}$ (Takara, Tokyo, Japan) in the ABI PRISM ${ }^{\circledR} 7500$ real-time PCR system (Applied Biosystems, Foster City, CA, USA). Real-time PCR was performed according to the manufacturer's instructions. Melting curves were used to evaluate non-specific amplification. All experiments were performed using biological triplicates and experimental duplicates. The relative expression levels were evaluated via the $2-\Delta \Delta \mathrm{Ct}$ method.

Western blotting. U87 or U251 cells were processed with RIPA lysis buffer (Saierbio, Tianjing, China) for $48 \mathrm{~h}$ before collecting protein samples. Total protein $(32 \mu \mathrm{l})$ and $8 \mu \mathrm{l}$ of loading buffer (5X) was boiled for $3 \mathrm{~min}$ and cooled down for $3 \mathrm{~min}$ on ice. Heat-denatured protein samples were resolved by $10 \%$ SDS-polyacrylamide gel (SDS-PAGE) electrophoresis under $100 \mathrm{~V}$ and transferred to hybond-nitrocellulose membranes (Millipore, USA) under $350 \mathrm{~mA}$ at $4^{\circ} \mathrm{C}$. The membrane was blocked in Blotto solution for $2 \mathrm{~h}$, and then incubated for $12 \mathrm{~h}$ at $4^{\circ} \mathrm{C}$ with primary rabbit anti-human monoclonal CELF2 antibody (1:1,000, Abcam, Cambridge, UK) overnight for combination. The GAPDH expression level was measured using rabbit anti-human monoclonal GAPDH antibody (1:3,000, Saier, Tianjing, China) as a loading control. Then the membrand was incubated for $2 \mathrm{~h}$ with HRP marked goat anti-rabbit second antibody (1:5,000, Saier, Tianjing, China). The bound antibody was detected with the use of Western Lightning ${ }^{\circledR}$-ECL, Enhanced Chemiluminescence Substrate (Perkin-Elmer, NEL100001EA). The band density of CELF2 was quantified after normalization with the density of GAPDH.

MTT assay. U87 $\left(1 \times 10^{4}\right)$ or U251 cells were seeded into each well of a 96-well plate one day before transfection. Following a 48-h incubation after transfection, $20 \mu \mathrm{l}$ of MTT reagent $(5 \mathrm{mg} / \mathrm{ml})$ was added into each well and the plate was incubated for another $4 \mathrm{~h}$. The supernatant was removed, and $150 \mu \mathrm{l}$ of DMSO was added into each well to dissolve the precipitate. The absorbance value was recorded at $570 \mathrm{~nm}$ using a microplate reader (BioTek, Winooski, VT, USA). All experiments were performed using biological triplicates and experimental duplicates, and the data are presented as mean \pm SD.

Flow cytometry. The apoptosis analysis was performed with Annexin V/7-AAD apoptosis detection kit (KeyGen, Nanjing, China) according to the manufacturer's instructions. Briefly, $5 \times 10^{5}$ cells were planted into a 6 -well plate and treated with 
$0.1 \%$ trypsin and $0.02 \%$ EDTA, and were washed twice with cold PBS before collection. The cells were mixed with $100 \mu \mathrm{l}$ of $1 \mathrm{X}$ binding buffer and resuspended. Annexin V (10 $\mu \mathrm{l})$ was added and incubated for $30 \mathrm{~min}$. Then $380 \mu \mathrm{l}$ of $1 \mathrm{X}$ binding buffer and $10 \mu \mathrm{l}$ of 7-AAD was added sequently and fully mixed with the cells. The fluorescence values of Annexin V and 7-AAD were measured by flow cytometry (Beckman Coulter, CA, USA).

For cell cycle assay, $5 \times 10^{5}$ cells were planted into a 6-well plate, washed by $1 \mathrm{X}$ PBS once and treated with $0.1 \%$ trypsin and $0.02 \%$ EDTA for digestion. The cells were suspended in $1 \mathrm{ml}$ culture medium and centrifuged under $12,000 \mathrm{x} \mathrm{g}$ for $5 \mathrm{~min}$. The supernatant was discarded and $1 \mathrm{ml} 1 \mathrm{X}$ PBS was added into the cells then centrifuged for $5 \mathrm{~min}$. The cells were stained with PI for $30 \mathrm{~min}$ and then investigate by flow cytometry. All the procedures were conducted according to the manufacturer's instructions.

Cell invasion assay. Cell invasion ability was determined by Corning transwell insert chambers (Corning, USA) and BD BioCoat Matrigel (BD Biosciences, Bedford, MA, USA). U87 or U251 cells were harvested and trypsinized in serum-free medium after transfection. Then the cell suspension $(200 \mu 1$, $1 \times 10^{5}$ cells) was added into the chamber and incubated $24 \mathrm{~h}$ at $37^{\circ} \mathrm{C}$. Cells that invaded the Matrigel and passed through the filter were fixed with $20 \%$ methanol for $30 \mathrm{~min}$, stained with crystal violet for $15 \mathrm{~min}$, and then imaged under x100 magnification Nikon 90I light microscope (Nikon, Japan) and counted in 6 randomized fields.

Vector construction. For overexpression of miR-95-3p, the human pre-miR-95 sequence was amplified and cloned into pcDNA3.1-his A constructs (Invitrogen) to generate pcDNA3.1-miR-95 expression vector. The primers were miR-95-KL-F: CCGGAATTCGAAGGTAGGATTGTGA CAC and miR-95-KL-R: CGCGGATCCGGAGGGATGGAT GAATGAC.

For luciferase reporter assay, the 3'UTR of the human CELF2 gene containing the putative binding site was PCR amplified using the following primers: wt-CELF2-Fw: AAA CTAGCGG CCGCTAGTCTAGGAATGGGCTTTTTTTCACCGTTGAA CCCTAGACCTGT; wt-CELF2-Rv: CTAGACAGGTCTAGG GTTCAACGGTGAAAAAAAGCCCATTCCTAGACTAGC GGCCGCTAGTTT. 3'UTR of CELF2 gene containing the mutant binding site used the following primers: mut-CELF2Fw: AAACTAGCGGCCGCTAGTCTAGGAATGGGCTTT TTTTCAGGCAACTACCCTAGACCTGT; mut-CELF2-Rv: CTAGACAGGTCTAGGGTAGTTGCCTGAAAAAAAGCC CATTCCTAGACTAGCGGCCGCTAGTTT. Fragments in CELF2 3'UTR containing the binding site (wt-CELF2) or mutated site (mut-CELF2) were, respectively, double-digested with $X b a \mathrm{I} / E c o$ RI and cloned downstream of firefly luciferase coding region sites of pmirGLO plasmid (Promega, Madison, WI, USA).

Luciferase reporter assay. The binding site was replaced with its complementary sequence to generate mutation. U251 cells were plated in a 96-well plate and then co-transfected with $0.25 \mu \mathrm{g}$ of wt-CELF2 or mut-CELF2 expression vector and $10 \mathrm{pmol}$ of miR-95-3p ASO or control ASO using Lipofectamine

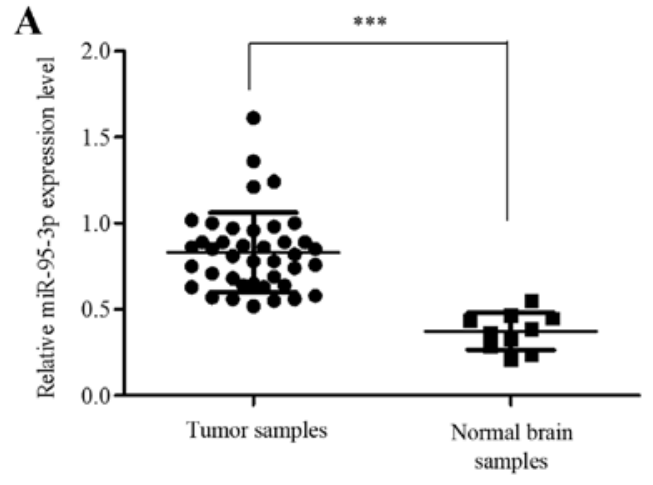

B

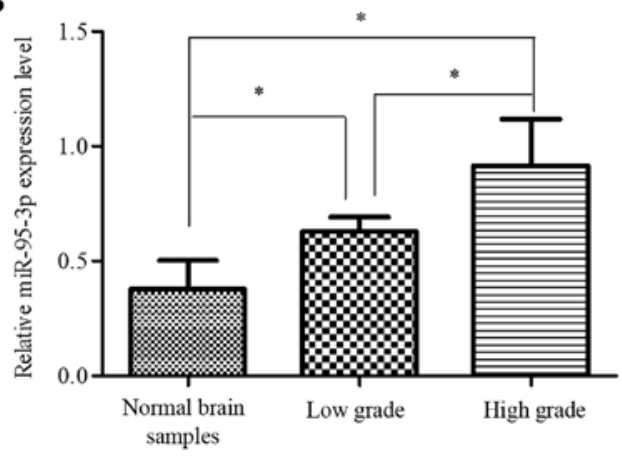

Figure 1. qRT-PCR assays of miR-95-3p expression level in glioma samples and non-neoplasm brain samples. (A) The level of miR-95-3p was significantly upregulated in glioma samples compared to that in non-neoplasm samples $\left.{ }^{* * *} \mathrm{P}<0.001\right)$. (B) The differences between non-neoplasm tissues, low grade glioma (grade I and II) and high grade glioma (grade III and IV) were significant $\left({ }^{*} \mathrm{P}<0.05\right)$.

2000 reagent. Cells were collected $48 \mathrm{~h}$ after transfection and analyzed using the Dual-Luciferase Reporter Assay System (Promega, Madison, WI, USA). Luciferase activity was detected by F200/M200 microplate fluorescence reader (Tecan Infinite). The co-transfected Renilla luciferase control vector was used as an internal control to correct the differences in both transfection and the harvesting efficiency. All the regents and instruments were performed according to the manufacturer's instructions. Transfection experiments were performed in duplicates and repeated at least thrice in independent experiments.

Statistical analysis. All tests and vector graphics were done using PRISM version 5.0 (GraphPad Software Inc., San Diego, CA, USA) statistical software. The experiments were carried out for three times independently. Data are mean \pm standard deviation except when indicated otherwise. Spearman's rank correlation test was used for association analysis between miR-95-3p and CELF2 level data. Student's t-test was used to analyze the statistical significance between two groups. One-way ANOVA was used to compare multiple groups. $\mathrm{P}<0.05$ was considered to be significant (shown in figures as: $\left.{ }^{*} \mathrm{P}<0.05 ;{ }^{* *} \mathrm{P}<0.01{ }^{* * *} \mathrm{P}<0.001\right)$.

\section{Results}

miR-95-3p is significantly upregulated in human glioma tissues. To determine the role of miR-95-3p in tumorigenesis of human glioma, we compared the expression level of miR-95-3p in glioma samples and non-neoplasma brain samples. In this study, 
A

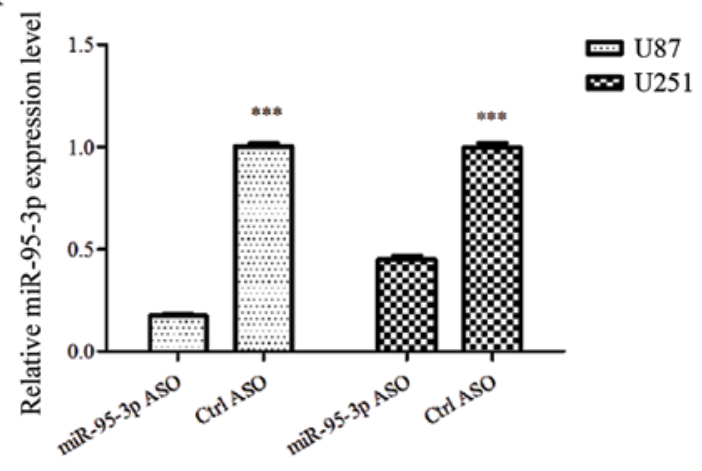

B

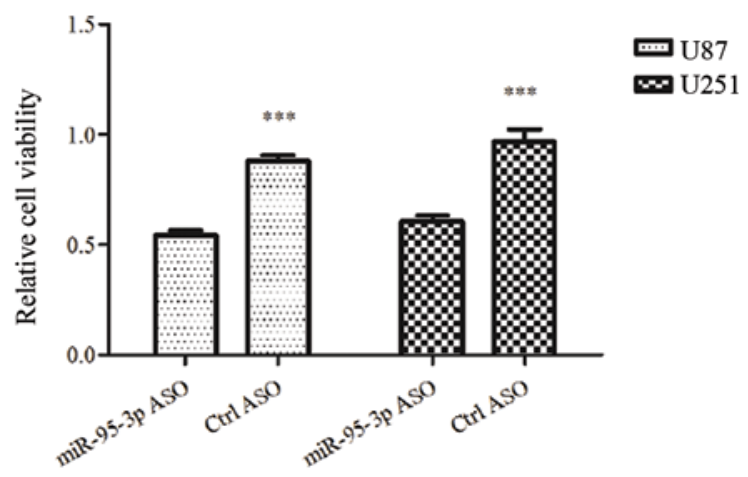

C
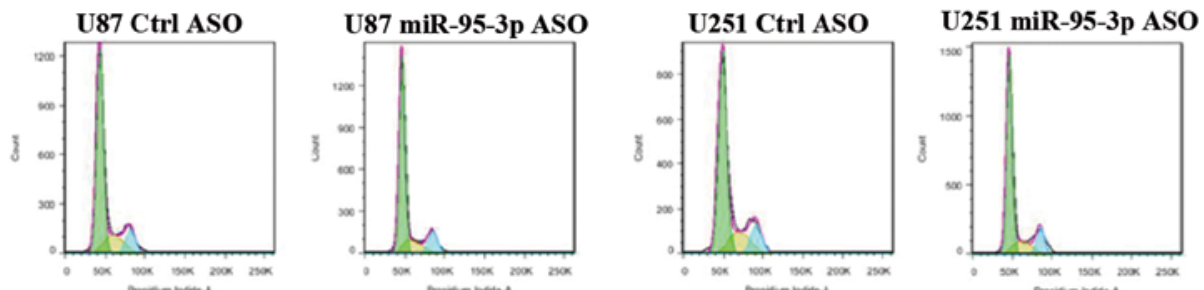

\begin{tabular}{|l|}
\hline Freq. $\mathrm{Gl}=60.07$ \\
Freq. $\mathrm{S}=17.95$ \\
Freq.G2=11.93 \\
\hline
\end{tabular}
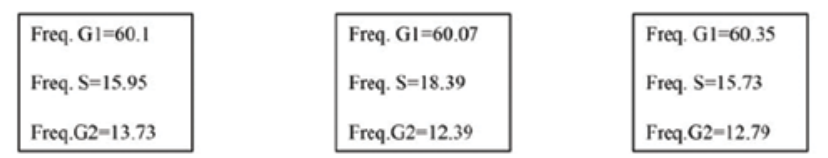

D
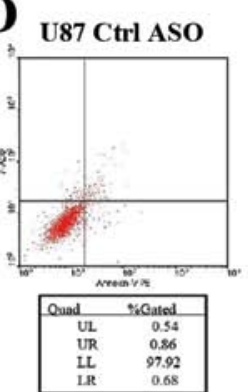

U251 Ctrl ASO
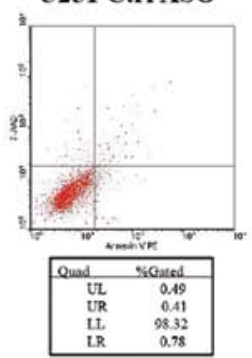
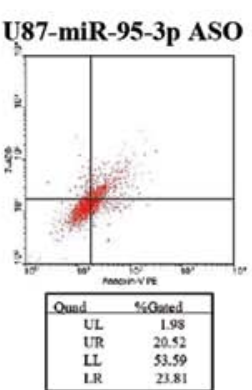

U251-miR-95-3p ASO
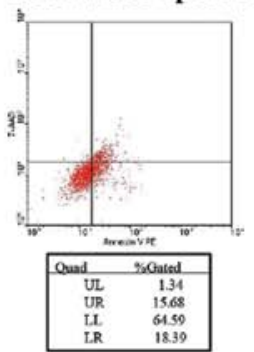

$\mathbf{E}$
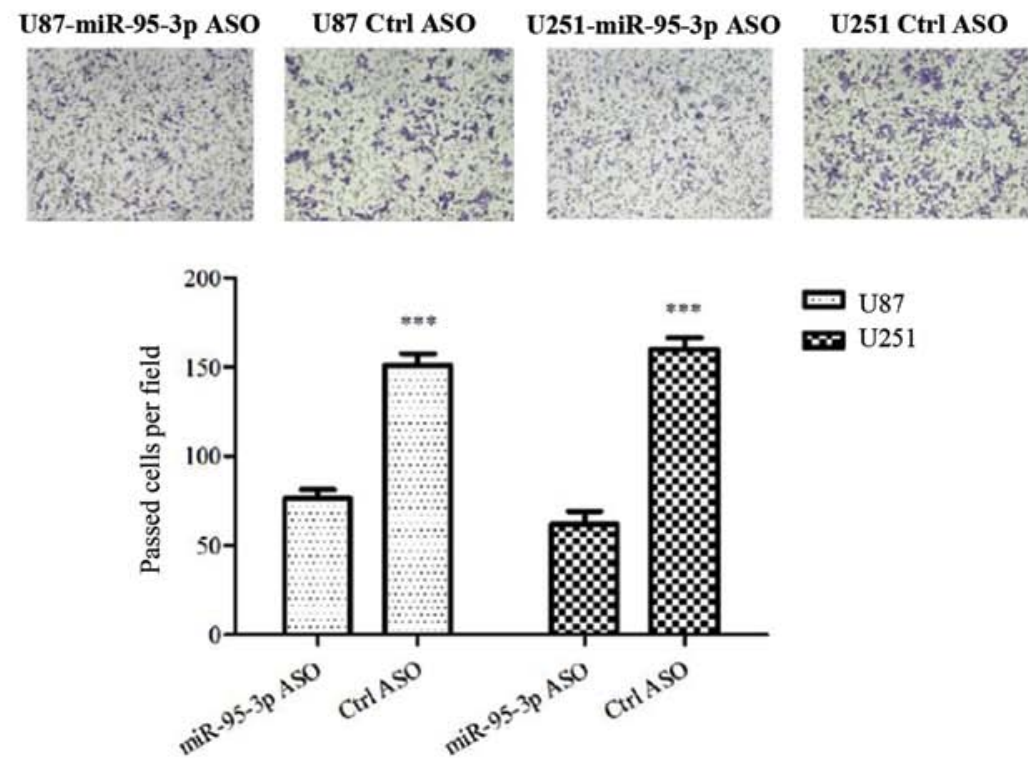

Figure 2. Evaluation of biological functions of miR-95-3p on U87 and U251 cells. (A) miR-95-3p expression level of experimental and control group in U87 and U251 cells by qRT-PCR. Data presented are mean values of three independent experimental results. ${ }^{* * *} \mathrm{P}<0.001$. (B) MTT assays confirmed that downregulation of miR-95-3p inhibited U87 or U251 cell proliferation, compared to control group. ${ }^{* * *} \mathrm{P}<0.001$. Relative absorbance is presented as mean \pm SD. (C) Cell cycle was determined by flow cytometry. Transfection of miR-95-3p ASO did not change the proportion of U87 or U251 cells in the different cell cycle phases significantly, compared to control group. (D) Cell apoptosis was determined by flow cytometry. In the panel, LL area represents active cells, while LR and UR areas represent apoptotic cells. Downregulation of miR-95-3p significantly promotes apoptosis in U87 or U251 cells, compared to control group. (E) The upper panel shows the cells invaded through the membrane observed by a light microscope (x100) after crystal violet staining. The lower panel shows the number of the cells invaded through the membrane. The images and number of cells were obtained in 6 randomized fields. U87 or U251 cells were decreased in invasive ability after being transfected with miR-95-3p ASO, compared to control group. ${ }^{* * *} \mathrm{P}<0.001$.

35 tumor samples were used as experimental group and 5 nonneoplasma samples as control. Case number, age, gender, WHO grade and pathological report of each patient were recorded.
The qRT-PCR results showed that miR-95-3p was significantly overexpressed in glioma tissues compared to control group (Fig. 1A). We also analyzed the correlation between expression 
A

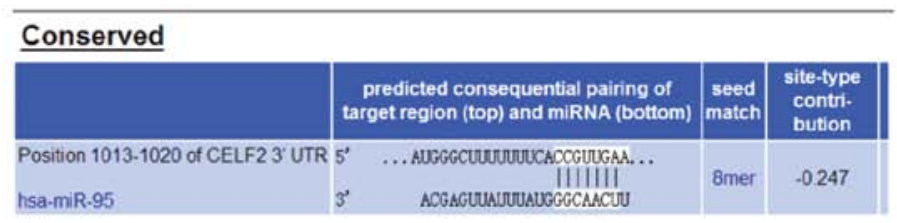

C

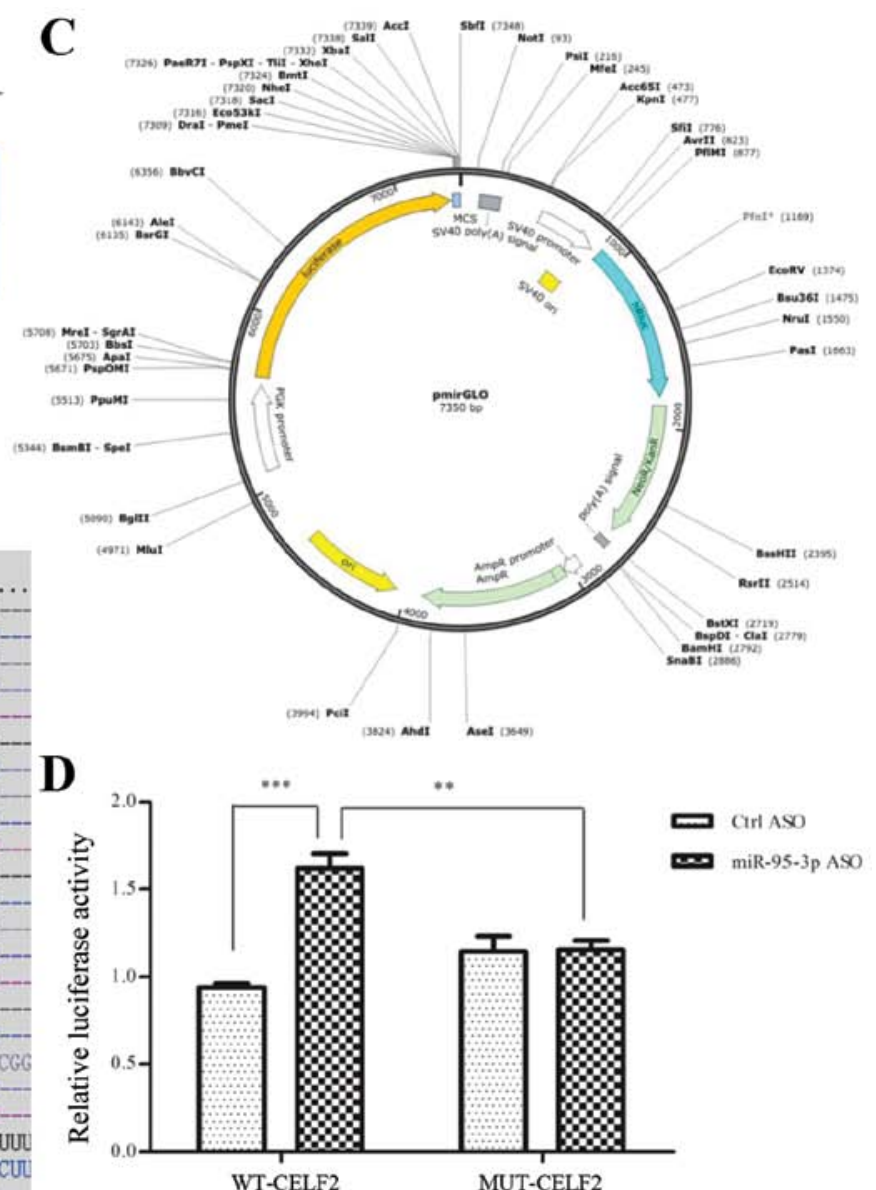

D

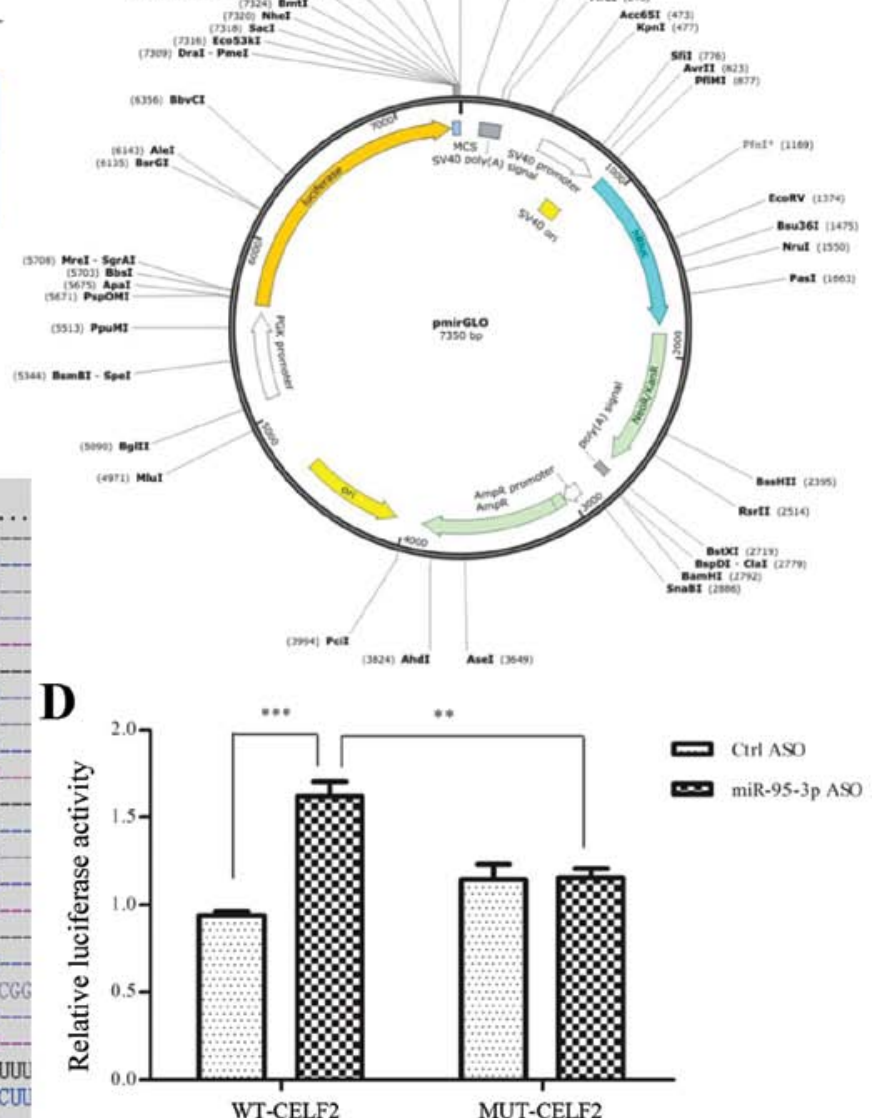

B

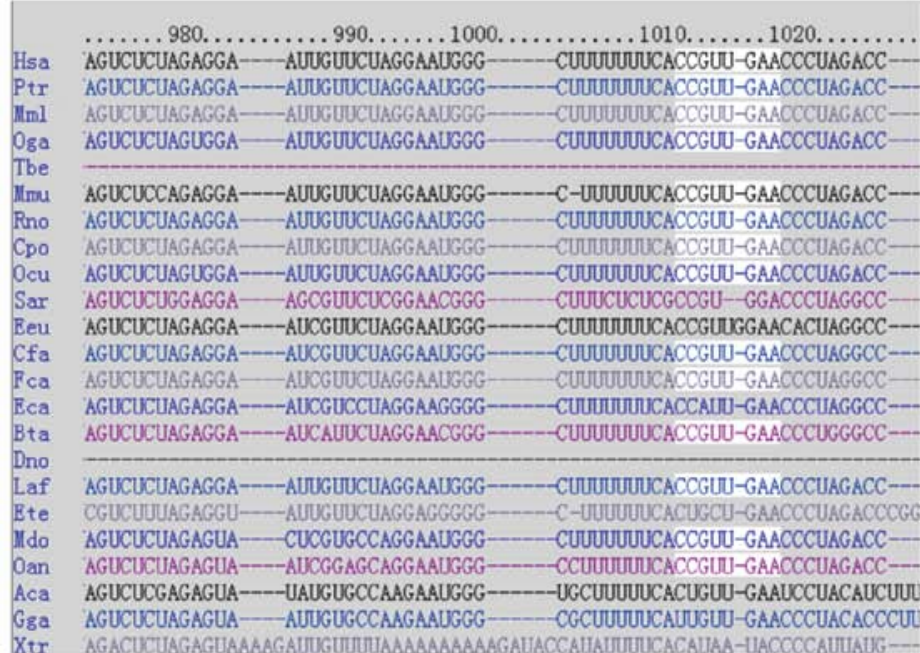

Figure 3. CELF2 is a bona fide target of miR-95-3p by luciferase assay. (A) Predicted binding sites of miR-95-3p in CELF2 3'UTR region by Targetscan (www.targetscan.org). (B) 3'UTR sequences of 14 different species recognized by miR-95-3p seed sequence in homology analysis, obtained from Targetscan (www.targetscan.org). (C) Mode pattern of pmirGLO vector for luciferase reporter assay. (D) miR-95-3p ASO could significantly increase luciferase activity in U251 cells transfected with wild-type CELF2 3'UTR, comparing to control ASO $\left({ }^{* * *} \mathrm{P}<0.0001\right)$. Mutation on binding sites significantly abrogated the effect of miR-95-3p ASO on CELF2 3'UTR, ${ }^{* *} \mathrm{P}<0.01$.

level and pathological grade. One-way ANOVA test showed that the differences between non-neoplasm tissues, low grade glioma (grade I and II) and high grade glioma (grade III and IV) were significant, while the differences between grade I and II or between grade III and IV were not (Fig. 1B).

Downregulation of miR-95-3p manifests tumor-suppressive functions in glioma cell lines. To understand the function of miR-95-3p in glioma cells, expression of miR-95-3p was downregulated in U87 and U251 cell lines for a loss-of-function behavior. Antisense oligonucleotide (ASO) was introduced into the cells to repress miR-95-3p expression. Control cells were transfected with false ASO. Forty-eight hours later, miR-95-3p expression levels were examined by qRT-PCR. As shown in Fig. 2A, miR-95-3p was downregulated in U87 and U251 cells after transfection of ASO. Then we investigated the nature of the cells with different miR-95-3p levels.

MTT assay was performed to investigate the effect of miR-95-3p on cell proliferation. We found that transfection of U87 cells with miR-95-3p ASO significantly decreased the relative absorbance of MTT, which was consistant with that of U251 cells (Fig. 2B). The data indicated an anti-proliferation effect of downregulation of miR-95-3p on glioma cells. Then, we conducted flow cytometry to analyze the effect of
miR-95-3p on the cell cycle and apoptosis. Our experiment failed to prove any difference in the cell cycle. The proportion of U87 or U251 cells in G1, S and G2 phase stayed almost unchanged after transfection with miR-95-3p ASO, compared to control group (Fig. 2C). As to apoptosis, U87 or U251 cells transfected with miR-95-3p ASO had a significant increase in number stained by Annexin $\mathrm{V}$ and/or 7-AAD, compared to cells with false ASO (Fig. 2D). The data showed that downregulation of miR-95-3p induces cell apoptosis. Finally, cell invasiveness was measured by transwell assay in order to evaluate metastasis potential. The cells were imaged and counted after $24 \mathrm{~h}$ of incubation in the transwell chamber. Our experiments showed less U87 or U251 cells transfected with miR-95-3p ASO invaded through the membrane than those with false ASO, which indicated downregulation of miR-95-3p decreased cell invasiveness (Fig. 2E). Taken together, the results manifested that downregulation of miR-95-3p has therapeutic effects on glioma proliferation, invasion and apoptosis.

CELF2 is a bona fide target of miR-95-3p. To delineate the mechanism by which miR-95-3p regulates the biological behavior of glioma cells, we searched the online program Targetscan for the target. Among all the protein candidates predicted, we finally recruited CELF2 (NM_001025077) 
A

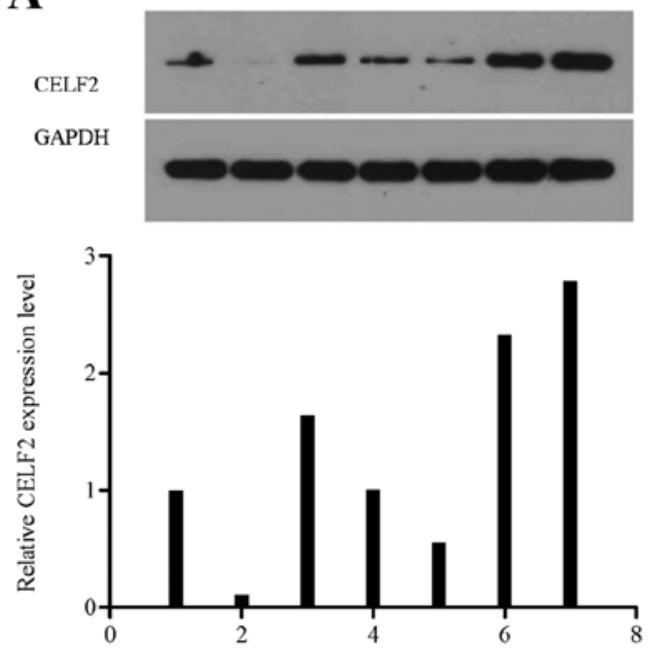

B

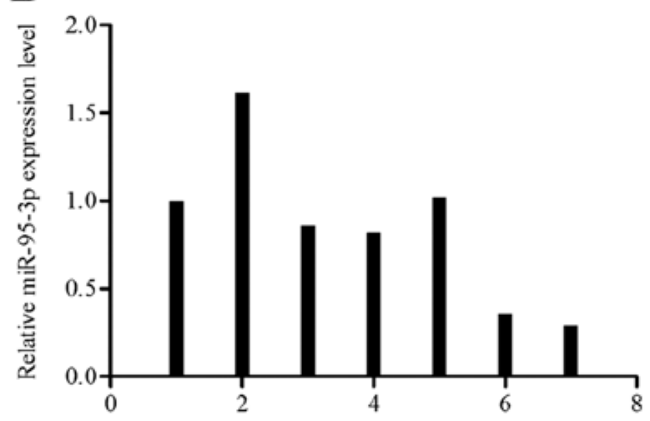

C

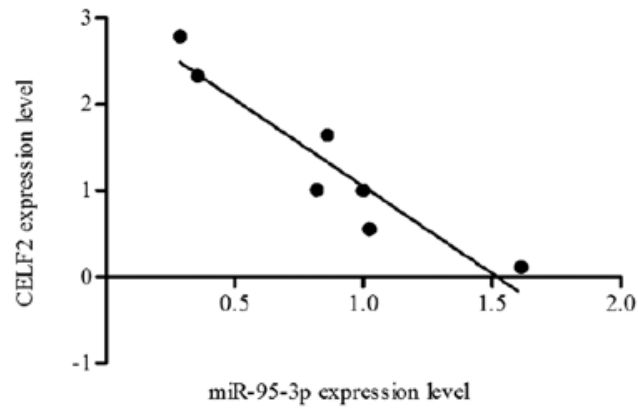

D
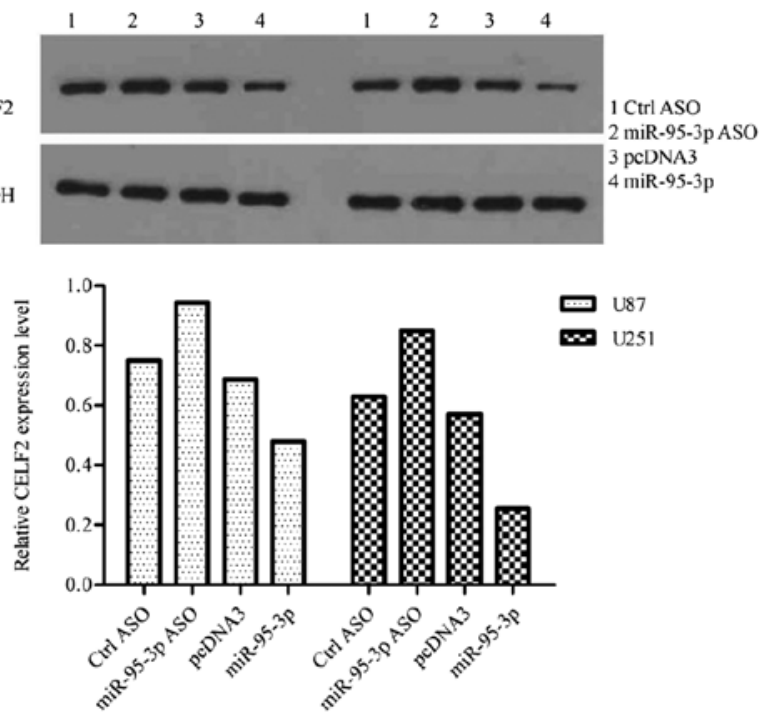

Figure 4. Expression relevance of CELF2 and miR-95-3p in glioma cells. (A) CELF2 expression profile in human tissues by western blotting and qRT-PCR (B) miR-95-3p expression profile in same human tissues by qRT-PCR. (C) A negative correlation exists between CELF2 and miR-95-3p expression level in human tissues. Spearman $r=-0.964{ }^{* *} \mathrm{P}<0.01$. (D) CELF2 was highly expressed after transfection of miR-95-3p ASO and low expressed after transfection of miR-95-3p expression vector in U87 and U251 cells, measured by qRT-PCR and western blot assay. GAPDH was used for normalization.

as a putative target and identified one potential binding site for miR-95-3p (Fig. 3A and B). To confirm that CELF2 is a bona fide target of miR-95-3p, luciferase reporter assays were performed. Fig. 3C shows the mode pattern of pmirGLO vector. We demonstrated the fact that miR-95-3p ASO could significantly increase luciferase activity, which could be abrogated by the mutation of the binding site (Fig. 3D).

We explored the expression profiles of CELF2 and miR-95-3p in 7 human samples. Each piece of sample used in this experiment for western blot assay came from the same tissue with that for qRT-PTR to make their results comparable (Fig. 4A and B). The results supported a negative correlation between CELF2 and miR-95-3p expression level in human tissues (Fig. 4C). We modified the level of miR-95-3p in U251 cells by tansfecting with ASO or expression vector, to investigate the effect of miR-95-3p on CELF2 in vitro. Compared to control cells, CELF2 was downregulated in cells overexpressing miR-95-3p while upregulated in cells low-expressing miR-95-3p (Fig. 4D). Based on the above, we proved that miR-95-3p suppresses CELF2 gene expression by regulating $3^{\prime} \mathrm{UTR}$ at the post-transcription level.

CELF 2 rescues the effect of miR-95-3p on glioma cells. To determine whether miR-95-3p regulates glioma cell biological behavior by targeting CELF2, CELF2 siRNA was used to perform rescue experiments. The downregulation of CELF2 by siRNA was validated by western blotting (Fig. 5A). The results showed that U251 cells transfected with miR-95-3p ASO plus CELF2 siRNA were increased in cell proliferation and invasion while decreased in apoptosis, compared to control (Fig. 5B-D). The data further suggest that CELF2 is a functional downstream target of miR-95-3p in regulating glioma biological behavior.

\section{Discussion}

Given the fact that cancer cells develop extraordinary characteristics which make them refractory to therapies, it is crucial to understand the underlying molecular biological mechanism. Evidence has shown miRNAs are implicated in many pathological and physiological processes, and we focused on miRNAs to explore their interaction with gliomas. The functions of miR-95-3p has been studied previously. Huang et al (22) found miR-95 could promote proliferation in human colorectal carcinoma cells. It has also been pointed out that miR-95 overexpression increased tumor growth and resistance to radiation treatment in prostate cancer cells (23). Our preliminary test showed that the expression difference of miR-95 was significant between glioma and non-neoplasm brain samples. 
A
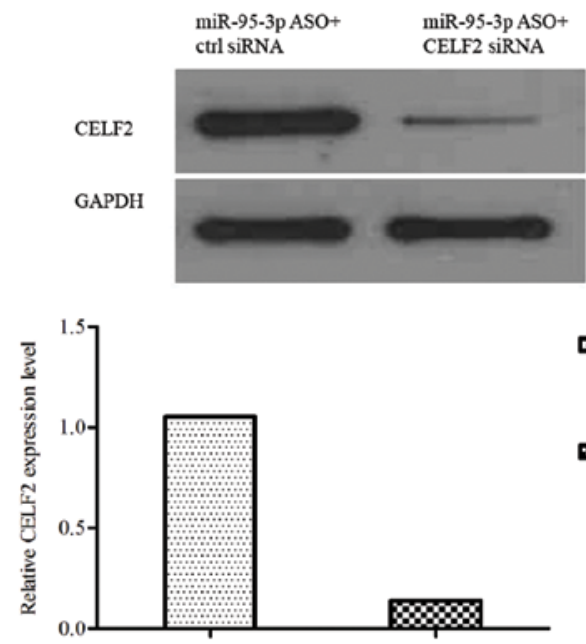

C

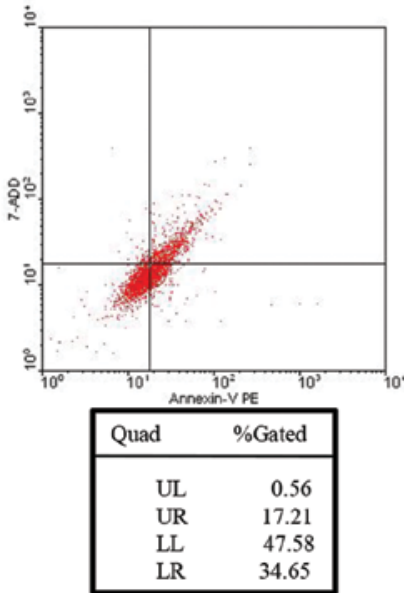

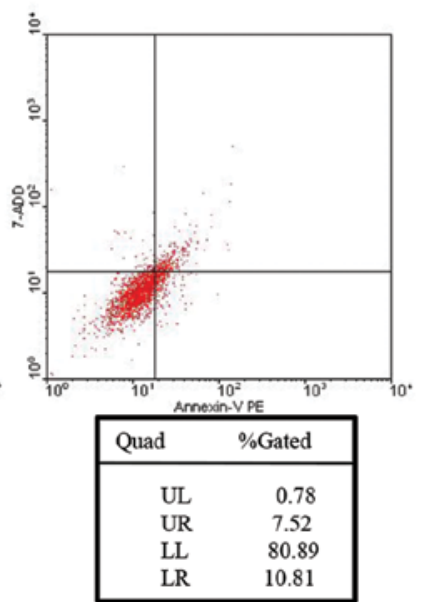

B

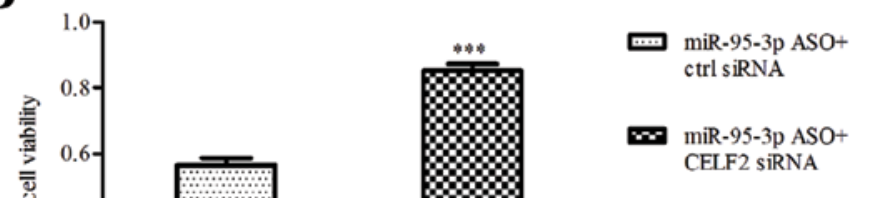

D
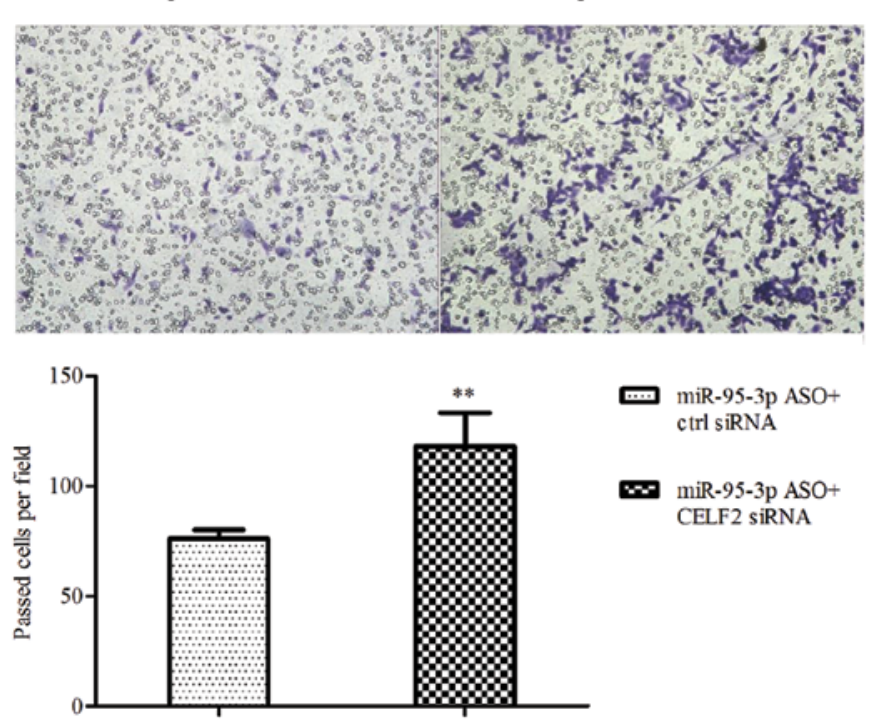

miR-95-3p ASO+ ctrl siRNA niR-95-3p ASO CELF2 siRNA

Figure 5. CELF2 reduces the effect of miR-95-3p on glioma cells. (A) The experimental group was transfected with miR-95-ASO+CELF2 siRNA and the control group with miR-95-ASO+Ctrl siRNA. Western blot assay showed CELF2 level was effectively repressed, compared to control. (B-D) The relative absorbance of MTT was increased $\left({ }^{* * *} \mathrm{P}<0.001\right)$, cell apoptosis was decreased and the number of cells invaded through the membrane was increased $(* * \mathrm{P}<0.01)$ compared to control. Images were taken at x100 magnification after crystal violet staining.

So we picked out miR-95-3p, one of miR-95 mature sequences, for study. We analyzed our data and tried to identify correlations of miR-95-3p expression level with pathological grade and found miR-95-3p level increased with WHO grade.

As miR-95-3p has hardly been investigated in glioma previously, we planned functional experiments on multiple aspects of tumorigenesis, including proliferation, cell cycle, apoptosis and invasion. In order to set up the experimental group, miR-95-3p ASO was transfected into glioma cells to reduce the miR-95-3p level. In MTT assay, we observed downregulation of miR-95-3p repressed glioma cells proliferation. MTT results reflected the change of mitochondria viability, or the amount of living cells, regardless of the cause. To explain how the living cells decreased, we further analyzed the cell cycle and apoptosis by flow cytometry. Our results manifested no significant difference in cell cycle between experimental and control group, which indicated miR-95-3p does not affect glioma cell growth rate. Apoptosis analysis showed a significantly increased number of cell death in the experimental group. So we inferred that miR-95-3p suppresses glioma cell proliferation by inducing apoptosis, rather than by slowing down cell mitosis. As invasiveness is an important factor in tumor metastasis, we also performed transwell test to investigate the function of miR-95-3p on this aspect. Less cells transfected with miR-95-3p invaded through the membrane than control, as we expected.

In the following phase of the study, we aimed to understand the mechanism by which miR-95-3p mediates glioma behavior. We searched the online programs TargetScan, mirWalk and PicTar to identify the target of miR-95-3p and focused on CELF2. CELF (CUGBP- and ETR-3-like family) is a group of RNA-binding proteins encoding various functions in post-transcriptional level, including alternative splicing, RNA editing and mRNA translation (27). These proteins contain 3 RNA recognition motifs (RRMs), which are 80-90 amino acid domains with conserve sequences for proteins to bind RNAs (28). Two of the RRMs are located near the $\mathrm{N}$-terminus and separated from the third one by an intervening bridge segment (29). CELF2 (also known as CUGBP2, NAPOR2, ETR3), one of the CELF family members, is expressed ubiquitously, albeit at higher level in mouse cells (30). In view of the fact that CELF2 was found involved in neuroblastoma cell apoptosis (31) and is encoded by a gene located on chromosome 10 , which shows 
monosomy in $\leq 60 \%$ and partial loss in $80 \%$ of high grade gliomas $(32,33)$, we hypothesized CELF2 may be implicated in the development of glioma as well. We employed luciferase reporter assay to verify our theory. The results indicated that miR-95-3p ASO significantly increased luciferase activity, which could be abrogated by mutation on the predicted binding site. In the experiments of western blotting and qRT-PCR, our data implied the levels of CELF2 and miR-95-3p were negatively correlated both in cell lines and human samples. The conclusion that CELF2 is a direct target of miR-95-3p was drawn.

To determine whether miR-95-3p regulates glioma cells biological behavior by CELF2, rescue experiments were performed. From the results we found that the effects of miR-95-3p ASO on glioma cells were rescued by decreased expression of CELF2. Thus, the miR-95-3p-CELF2 regulation mechanism on glioma was established. Furthermore, CELF2 was also reported to promote cell apoptosis by mediating Mcl-1, and downregulation of CELF2 by prostaglandin E2 represses mitotic catastrophe induced by radiation in colon cancer cells $(34,35)$. Subramaniam et al $(36)$ also found that CELF2 plays a role in mitotic catastrophe of pancreatic cancer cells. It can be inferred that CELF2 may play an important role in the mitotic process, although miR-95-3p does not, and there may be other pathways related to CELF2 regulating glioma.

In conclusion, this study demonstrates miR-95-3p is upregulated in glioma samples and downregulation of miR-95-3p inhibits proliferation, invasion and promotes apoptosis of glioma cells by targeting CELF2. Thus, we identified miR-95-3p as a putative therapeutic target and CELF2 as a potential tumor suppressor.

\section{Acknowledgements}

We thank Mr. Shuang Chen and Yong Pu (Saierbio, Tianjing, China) for their technical advice.

\section{References}

1. Giese A, Bjerkvig R, Berens ME and Westphal M: Cost of migration: Invasion of malignant gliomas and implications for treatment. J Clin Oncol 21: 1624-1636, 2003.

2. Lefranc F, Brotchi J and Kiss R: Possible future issues in the treatment of glioblastomas: Special emphasis on cell migration and the resistance of migrating glioblastoma cells to apoptosis. J Clin Oncol 23: 2411-2422, 2005.

3. Singh SK, Clarke ID, Hide T and Dirks PB: Cancer stem cells in nervous system tumors. Oncogene 23: 7267-7273, 2004.

4. Furnari FB, Fenton T, Bachoo RM, Mukasa A, Stommel JM, Stegh A, Hahn WC, Ligon KL, Louis DN, Brennan C, et al: Malignant astrocytic glioma: Genetics, biology, and paths to treatment. Genes Dev 21: 2683-2710, 2007.

5. Bartel DP: MicroRNAs: Genomics, biogenesis, mechanism, and function. Cell 116: 281-297, 2004.

6. Eulalio A, Huntzinger E and Izaurralde E: Getting to the root of miRNA-mediated gene silencing. Cell 132: 9-14, 2008.

7. Carthew RW: Gene regulation by microRNAs. Curr Opin Genet Dev 16: 203-208, 2006.

8. Zhang B, Pan X, Cobb GP and Anderson TA: microRNAs as oncogenes and tumor suppressors. Dev Biol 302: 1-12, 2007.

9. Lujambio A and Lowe SW: The microcosmos of cancer. Nature 482: 347-355, 2012.

10. Bagnoli M, De Cecco L, Granata A, Nicoletti R, Marchesi E, Alberti P, Valeri B, Libra M, Barbareschi M, Raspagliesi F, et al: Identification of a chrXq27.3 microRNA cluster associated with early relapse in advanced stage ovarian cancer patients. Oncotarget 2: 1265-1278, 2011.
11. Iorio MV and Croce CM: microRNA involvement in human cancer. Carcinogenesis 33: 1126-1133, 2012.

12. Godlewski J, Nowicki MO, Bronisz A, Nuovo G, Palatini J, De Lay M, Van Brocklyn J, Ostrowski MC, Chiocca EA and Lawler SE: MicroRNA-451 regulates LKB1/AMPK signaling and allows adaptation to metabolic stress in glioma cells. Mol Cell 37: 620-632, 2010.

13. Gabriely G, Yi M, Narayan RS, Niers JM, Wurdinger T, Imitola J, Ligon KL, Kesari S, Esau C, Stephens RM, et al: Human glioma growth is controlled by microRNA-10b. Cancer Res 71: 3563-3572, 2011.

14. Li Y, Guessous F, Zhang Y, Dipierro C, Kefas B, Johnson E, Marcinkiewicz L, Jiang J, Yang Y, Schmittgen TD, et al: MicroRNA-34a inhibits glioblastoma growth by targeting multiple oncogenes. Cancer Res 69: 7569-7576, 2009.

15. Chan JA, Krichevsky AM and Kosik KS: MicroRNA-21 is an antiapoptotic factor in human glioblastoma cells. Cancer Res 65: 6029-6033, 2005.

16. Ciafrè SA, Galardi S, Mangiola A, Ferracin M, Liu CG, Sabatino G, Negrini M, Maira G, Croce CM and Farace MG: Extensive modulation of a set of microRNAs in primary glioblastoma. Biochem Biophys Res Commun 334: 1351-1358, 2005.

17. Zhou J, Wang W, Gao Z, Peng X, Chen X, Chen W, Xu W, Xu H, Lin MC and Jiang S: MicroRNA-155 promotes glioma cell proliferation via the regulation of MXI1. PLoS One 8: e83055, 2013.

18. Godlewski J, Nowicki MO, Bronisz A, Williams S, Otsuki A, Nuovo G, Raychaudhury A, Newton HB, Chiocca EA and Lawler S: Targeting of the Bmi-1 oncogene/stem cell renewal factor by microRNA-128 inhibits glioma proliferation and selfrenewal. Cancer Res 68: 9125-9130, 2008.

19. Silber J, Lim DA, Petritsch C, Persson AI, Maunakea AK, Yu M, Vandenberg SR, Ginzinger DG, James CD, Costello JF, et al: miR-124 and miR-137 inhibit proliferation of glioblastoma multiforme cells and induce differentiation of brain tumor stem cells. BMC Med 6: 14, 2008.

20. Bier A, Giladi N, Kronfeld N, Lee HK, Cazacu S, Finniss S, Xiang C, Poisson L, deCarvalho AC, Slavin S, et al: MicroRNA-137 is downregulated in glioblastoma and inhibits the stemness of glioma stem cells by targeting RTVP-1. Oncotarget 4: 665-676, 2013.

21. Skalsky RL and Cullen BR: Reduced expression of brain-enriched microRNAs in glioblastomas permits targeted regulation of a cell death gene. PLoS One 6: e24248, 2011.

22. Huang Z, Huang S, Wang Q, Liang L, Ni S, Wang L, Sheng W, $\mathrm{He} X$ and Du X: MicroRNA-95 promotes cell proliferation and targets sorting Nexin 1 in human colorectal carcinoma. Cancer Res 71: 2582-2589, 2011.

23. Huang X, Taeb S, Jahangiri S, Emmenegger U, Tran E, Bruce J, Mesci A, Korpela E, Vesprini D, Wong CS, et al: miRNA-95 mediates radioresistance in tumors by targeting the sphingolipid phosphatase SGPP1. Cancer Res 73: 6972-6986, 2013.

24. Zhang Y, Li M, Wang H, Fisher WE, Lin PH, Yao Q and Chen C: Profiling of 95 microRNAs in pancreatic cancer cell lines and surgical specimens by real-time PCR analysis. World J Surg 33: 698-709, 2009.

25. Xiao Z, Ching Chow S, Han Li C, Chun Tang S, Tsui SK, Lin Z and Chen Y: Role of microRNA-95 in the anticancer activity of Brucein D in hepatocellular carcinoma. Eur J Pharmacol 728: 141-150, 2014.

26. Nurul-Syakima AM, Yoke-Kqueen C, Sabariah AR, Shiran MS, Singh A and Learn-Han L: Differential microRNA expression and identification of putative miRNA targets and pathways in head and neck cancers. Int J Mol Med 28: 327-336, 2011.

27. Barreau C, Paillard L, Méreau A and Osborne HB: Mammalian CELF/Bruno-like RNA-binding proteins: Molecular characteristics and biological functions. Biochimie 88: 515-525, 2006.

28. Keene JD: RNA recognition by autoantigens and autoantibodies. Mol Biol Rep 23: 173-181, 1996.

29. Ladd AN and Cooper TA: Multiple domains control the subcellular localization and activity of ETR-3, a regulator of nuclear and cytoplasmic RNA processing events. J Cell Sci 117: 3519-3529, 2004.

30. Wang GS, Kearney DL, De Biasi M, Taffet G and Cooper TA: Elevation of RNA-binding protein CUGBP1 is an early event in an inducible heart-specific mouse model of myotonic dystrophy. J Clin Invest 117: 2802-2811, 2007.

31. Choi DK, Ito T, Mitsui Y and Sakaki Y: Fluorescent differential display analysis of gene expression in apoptotic neuroblastoma cells. Gene 223: 21-31, 1998. 
32. Pershouse MA, Stubblefield E, Hadi A, Killary AM, Yung WK and Steck PA: Analysis of the functional role of chromosome 10 loss in human glioblastomas. Cancer Res 53: 5043-5050, 1993.

33. Ransom DT, Ritland SR, Moertel CA, Dahl RJ, O'Fallon JR, Scheithauer BW, Kimmel DW, Kelly PJ, Olopade OI, Diaz MO, et al: Correlation of cytogenetic analysis and loss of heterozygosity studies in human diffuse astrocytomas and mixed oligo-astrocytomas. Genes Chromosomes Cancer 5: 357-374, 1992.

34. Subramaniam D, Natarajan G, Ramalingam S, Ramachandran I, May R, Queimado L, Houchen CW and Anant S: Translation inhibition during cell cycle arrest and apoptosis: Mcl-1 is a novel target for RNA binding protein CUGBP2. Am J Physiol Gastrointest Liver Physiol 294: G1025-G1032, 2008.
35. Natarajan G, Ramalingam S, Ramachandran I, May R, Queimado L, Houchen CW and Anant S: CUGBP2 downregulation by prostaglandin E2 protects colon cancer cells from radiation-induced mitotic catastrophe. Am J Physiol Gastrointest Liver Physiol 294: G1235-G1244, 2008.

36. Subramaniam D, Ramalingam S, Linehan DC, Dieckgraefe BK, Postier RG, Houchen CW, Jensen RA and Anant S: RNA binding protein CUGBP2/CELF2 mediates curcumin-induced mitotic catastrophe of pancreatic cancer cells. PLoS One 6: e16958, 2011. 